\title{
A Rare Case of Duodenal Carcinoid Tumors in a Patient With Atypical Symptoms
}

\author{
Tanisha Wandhare ${ }^{\mathrm{a}, \mathrm{c}}$, Manrique Guerrero ${ }^{\mathrm{b}}$, Robert Madlinger ${ }^{\mathrm{b}}$, Jamshed Zuberi ${ }^{\mathrm{b}}$
}

\begin{abstract}
Gastrointestinal neuroendocrine tumors (NETs) are a common, yet relatively less understood subset of gastrointestinal tumors with a myriad of clinical presentations and prognostic outcomes. Although most NETs are more indolent than carcinomas, prognostic outcomes are still heavily reliant on several variables such as tumor location, stage, differentiation and metastasis amongst others. Additionally, NETs also present with a range of clinical symptoms, both localized and systemic. In this case report we discuss a patient, who had a longstanding symptom at presentation and was found to have a duodenal polyp upon endoscopy. Further evaluation established the presence of a well differentiated NET that was later resected surgically.
\end{abstract}

Keywords: Duodenal carcinoid; Carcinoid tumor; Carcinoid syndrome; Neuroendocrine tumors; Gastrointestinal NETs

\section{Introduction}

Neuroendocrine tumors (NETs) are a subset of gastrointestinal tumors that have seen an increase in incidence and detection in the past few decades. According to the Surveillance, Epidemiology, and End Results (SEER) program, NETs have had a five-fold increase in detection from 1973 to 2004, and are diagnosed at a median age of 63 years, with a higher incidence in males and African Americans [1].

About $19 \%$ of NETs can be associated with carcinoid syndrome, with characteristic symptoms such as flushing, diarrhea, nausea, vomiting, abdominal pain and wheezing [2]. These symptoms are caused by the local secretion of vasoactive amines such as serotonin and histamine. Carcinoid syndrome is more commonly seen in well differentiated NETs as well as regionally advanced tumors [3]. A potentially critical and life-threatening complication of carcinoid syndrome, known as carcinoid crisis can occur in patients in the set-

Manuscript submitted November 20, 2019, accepted December 5, 2019

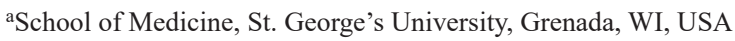

bSt. Joseph's University Medical Center, Paterson, NJ, USA

'Corresponding Author: Tanisha Wandhare, School of Medicine, St. George's

University, Grenada, WI, USA. Email: twandhar@sgu.edu

doi: https://doi.org/10.14740/jcs397 ting of stressors such as anesthesia and surgery. The crisis is characterized by excessive release of vasoactive amines into systemic circulation causing hypotension, tachycardia, arrhythmias and bronchospasm [4]. Most NETs occur in the gastrointestinal tract $(54.5 \%)$, with the most predominant site being the ileum $(>50 \%)$. Within the gastrointestinal tract, the duodenum is the rarest location for carcinoid tumors to occur [5]. Based on the data from the SEER program, duodenal NETs comprise only about $1-3 \%$ of primary duodenal tumors, with an overall incidence of $0.19 / 100,000$ in the USA, and only five case studies have described patients with duodenal carcinoids [1].

\section{Case Report}

A 52-year-old Caucasian female presented to the emergency room with epigastric abdominal pain for the past 1 day and 15 episodes of vomiting of undigested stomach contents over the course of a few hours. The patient had previously experienced several similar episodes of abdominal pain, nausea and vomiting over the duration of 1 year. These episodes would last for a few days and resolve with over the counter pain relieving medication. Of note, the patient had a past medical history significant for diastolic heart failure, insulin dependent diabetes mellitus, hypertension, and coronary artery disease.

\section{Investigations}

The patient had a previous hospital admission 2 weeks prior for similar symptoms and underwent esophagoduodenoscopy (EGD) at the time. The EGD showed a duodenal bulb polyp (Fig. 1) and antral gastritis. Preoperative computed tomography (CT) scans showing cardiomegaly, hepatomegaly, multiple shotty mesenteric, periaortic aortocaval, periportal, gastrohepatic ligament lymph nodes, fullness of both adrenal glands, left lower pole renal cyst, aortoiliac atherosclerosis, and shotty bilateral inguinal lymph nodes (Fig. 2a, b).

\section{Differential diagnosis}

Surgical pathology report on the biopsy indicated a well differentiated neuroendocrine (carcinoid) tumor, with cells positive for synaptophysin and chromogranin. The patient was 


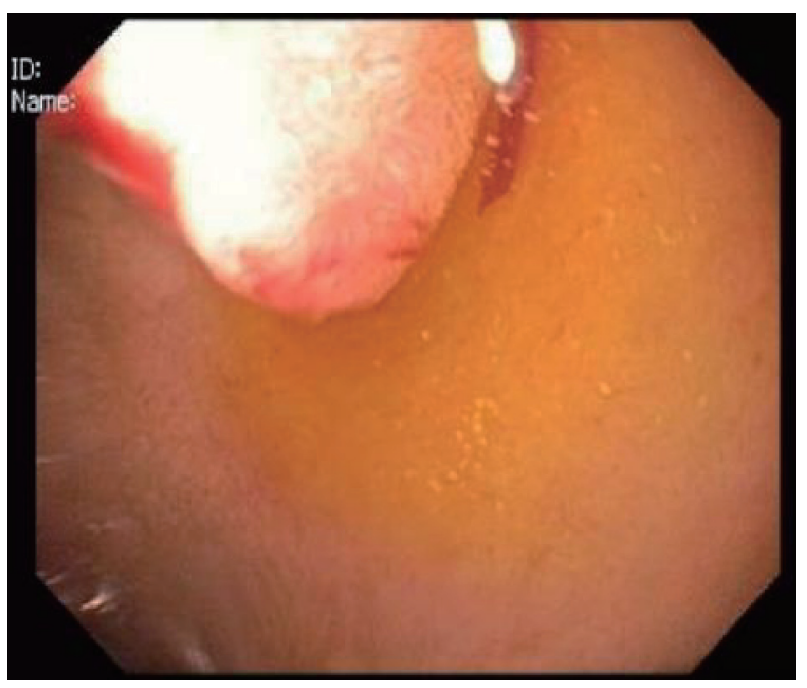

Figure 1. Bulbar polyp seen on EGD. EGD: esophagoduodenoscopy.

discharged and advised to undergo an EGD/endoscopic ultrasound (EUS) for polypectomy.

\section{Treatment}

Given the patient's long standing history of symptoms and previous findings of the EGD the patient was admitted and underwent an exploratory laparotomy for excision of the carcinoid tumor. During exploration she was found to have a second tumor at the pylorus. Both tumors were excised after a duodenostomy and sent for frozen section during the surgery. A Penrose drain was placed in the Morison's pouch. Air insufflation and methylene blue flush showed no leaks from the duodenal repair and pyloroplasty.

\section{Outcome and follow-up}

The patient was shifted to the surgical intensive care unit (ICU) for monitored management of her pre-existing medical conditions and discharged 2 weeks later.

\section{Discussion}

The incidence of carcinoid syndrome in patients with carcinoid tumors varies greatly based on the location of the tumors. Based on the SEER database, several studies have demonstrated that typical features of the carcinoid syndrome were not common in duodenal, rectal, central nervous system (CNS), type 1 gastric, colonic or appendiceal carcinoids, while NETs in other areas of the small intestine, pulmonary tract, pancreas, type 3 gastric carcinoids and Meckel's diverticulum were eight times more likely to present with symptoms such as diarrhea, nausea, wheezing, cutaneous flushing, hyperpigmentation, and hyperkeratosis. Several uncommon yet more morbid manifestations of carcinoid syndrome include carcinoid heart disease (CHD), rheumatoid arthritis, intestinal/mesenteric vessel fibrosis, retroperitoneal fibrosis, and pleural/pulmonary fibrosis [4].

The pathophysiology behind the typical features of the syndrome is vasoactive mediators such as serotonin, tachykinins, prostaglandins and in foregut carcinoids, histamine [3]. However the increased production of serotonin by the enterochromaffin cells of carcinoid tumors also leads to the proliferation of myofibroblasts and the increased deposition of extracellular matrix (collagen, elastin etc.) which has been known to contribute to the development of pulmonary, endovascular and endocardial fibrosis. Patients with carcinoid heart disease often present with tricuspid stenosis/regurgitation, but they may also have concurrent left-sided valvular disease, although less commonly [6]. Although our patient did not exhibit all the typical symptoms of carcinoid syndrome and did not have liver metastasis on presentation, her comorbidities of hypertension and diastolic heart failure may indicate the systemic effects of serotonin seen in as many as $35-59 \%$ of patients with carcinoid tumors [7].

The most common treatment for non-metastatic carcinoid tumors is surgical excision. A study of 1,914 cases of gastrointestinal carcinoids taken from the Niigata Registry showed that about $68 \%$ of the patients with submucosal NETs underwent a wider resection of the tumors, while $25 \%$ of the tumors were resected endoscopically [8]. A placebo controlled trial of octreotide in 90 patients with metastatic or inoperable NETs showed that somatostatin analogues were effective in controlling tumor growth in treatment naive patients and in patients with prior partial resections and low hepatic tumor load [9].
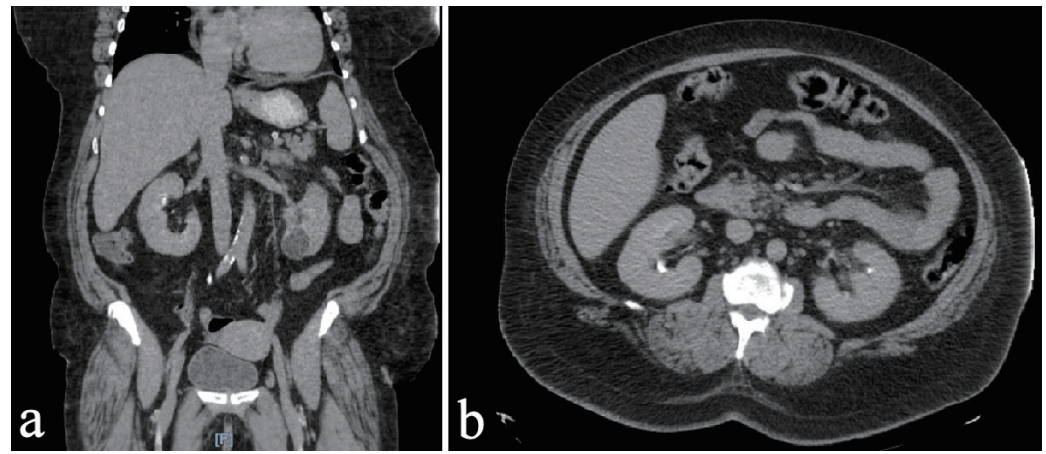

Figure 2. Preoperative CT scans. Preoperative CT scans showing cardiomegaly, hepatomegaly, multiple shotty mesenteric, periaortic aortocaval, periportal, gastrohepatic ligament lymph nodes, fullness of both adrenal glands, left lower pole renal cyst, aortoiliac atherosclerosis, and shotty bilateral inguinal lymph nodes. CT: computed tomography. 
Although most NETs are more indolent than carcinomas, prognostic outcomes are still heavily reliant on several variables such as tumor location, stage, differentiation and metastasis amongst others [10]. This makes NETs important subjects of investigation focusing on sensitive diagnosis, effective treatment, post-treatment follow-up, long-term prognosis, and patient quality of life.

\section{Conclusions}

Gastrointestinal NETs are a common, yet relatively less understood subset of gastrointestinal tumors with a myriad of clinical presentations and prognostic outcomes. NETs also present with a range of clinical symptoms, both localized and systemic. Duodenal carcinoid tumors are an extremely rare subset of NETs. As seen in our patient, even in the absence of radiologically evident hepatic metastasis, duodenal carcinoid tumors can be linked to multiple comorbidities such as carcinoid heart disease. NETs are indolent and therefore effective diagnoses and early intervention can help prevent severe systemic effects of carcinoid syndrome in patients.

\section{Acknowledgments}

None to declare.

\section{Financial Disclosure}

None to declare.

\section{Conflict of Interest}

None to declare.

\section{Informed Consent}

Not applicable.

\section{Author Contributions}

TW participated in the case and wrote the case report. MG, RM and JZ edited the case report.

\section{Data Availability}

Any inquiries regarding supporting data availability of this study should be directed to the corresponding author.

\section{References}

1. Yao JC, Hassan M, Phan A, Dagohoy C, Leary C, Mares JE, Abdalla EK, et al. One hundred years after "carcinoid": epidemiology of and prognostic factors for neuroendocrine tumors in 35,825 cases in the United States. J Clin Oncol. 2008;26(18):3063-3072.

2. Halperin DM, Shen C, Dasari A, Xu Y, Chu Y, Zhou S, Shih YT, et al. Frequency of carcinoid syndrome at neuroendocrine tumour diagnosis: a population-based study. Lancet Oncol. 2017;18(4):525-534.

3. Cives M, Strosberg JR. Gastroenteropancreatic neuroendocrine tumors. CA Cancer J Clin. 2018;68(6):471-487.

4. Rubin de Celis Ferrari AC, Glasberg J, Riechelmann RP. Carcinoid syndrome: update on the pathophysiology and treatment. Clinics (Sao Paulo). 2018;73(suppl 1):e490s.

5. Maggard MA, O'Connell JB, Ko CY. Updated population-based review of carcinoid tumors. Ann Surg. 2004;240(1):117-122.

6. Ito T, Lee L, Jensen RT. Carcinoid-syndrome: recent advances, current status and controversies. Curr Opin Endocrinol Diabetes Obes. 2018;25(1):22-35.

7. Mota JM, Sousa LG, Riechelmann RP. Complications from carcinoid syndrome: review of the current evidence. Ecancermedicalscience. 2016;10:662.

8. Soga J. Early-stage carcinoids of the gastrointestinal tract: an analysis of 1914 reported cases. Cancer. 2005;103(8):1587-1595.

9. Rinke A, Muller HH, Schade-Brittinger C, Klose KJ, Barth P, Wied M, Mayer C, et al. Placebo-controlled, double-blind, prospective, randomized study on the effect of octreotide LAR in the control of tumor growth in patients with metastatic neuroendocrine midgut tumors: a report from the PROMID Study Group. J Clin Oncol. 2009;27(28):4656-4663.

10. Garcia-Carbonero R, Capdevila J, Crespo-Herrero G, Diaz-Perez JA, Martinez Del Prado MP, Alonso Orduna $\mathrm{V}$, Sevilla-Garcia I, et al. Incidence, patterns of care and prognostic factors for outcome of gastroenteropancreatic neuroendocrine tumors (GEP-NETs): results from the National Cancer Registry of Spain (RGETNE). Ann Oncol. 2010;21(9):1794-1803. 